\title{
Investigating graphical representations of slope and derivative without a physics context
}

\author{
Warren M. Christensen ${ }^{1}$ and John R. Thompson ${ }^{2,3}$ \\ ${ }^{1}$ Department of Physics, North Dakota State University, Fargo, North Dakota 58108, USA \\ ${ }^{2}$ Department of Physics and Astronomy, University of Maine, Orono, Maine 04469, USA \\ ${ }^{3}$ Maine Center for Research in STEM Education, University of Maine, Orono, Maine 04469, USA
}

(Received 16 May 2011; published 26 July 2012)

\begin{abstract}
By analysis of student use of mathematics in responses to conceptual physics questions, as well as analogous math questions stripped of physical meaning, we have previously found evidence that students often enter upper-level physics courses lacking the assumed prerequisite mathematics knowledge and/or the ability to apply it productively in a physics context. As an extension from this work on students' mathematical competency at the upper level in physics, we report on a preliminary investigation of mathematical understanding of fundamental concepts of slope and derivative among students in a thirdsemester multivariable calculus course. Among the first published findings of physics education research are investigations on students' understanding of kinematics, with particular attention to graphical representations of position-, velocity-, and acceleration-versus-time graphs. Underlying these physical quantities are relationships that depend on derivatives and slopes. We report on our findings as we attempt to isolate students' understanding of these mathematical concepts.
\end{abstract}

DOI: 10.1103/PhysRevSTPER.8.023101

PACS numbers: $01.40 .-\mathrm{d}$

\section{INTRODUCTION}

Among the earliest findings in the physics education research (PER) literature are those difficulties reported by Trowbridge and McDermott concerning student understanding of kinematics [1,2]. A significant portion of this work was done through the analysis of student ideas about graphical representations of various kinematic processes and was followed a few years later by the work of McDermott et al. [3], which further analyzed student thinking of these concepts. A decade later, Beichner developed a multiple-choice survey to assess student knowledge of graphical representations in the context of kinematics called the Test of Understanding GraphsKinematics (TUG-K) [4]. Beichner's results corroborated findings previously reported by McDermott et al., including "slope/height/area confusion" in the context of kinematics among students in the introductory physics sequences.

The work we present here grew out of a broader study on the learning and teaching of thermal physics, dealing with identifying and addressing student conceptual difficulties with the physics content. A major subtheme of our research into students' understanding of thermal physics involves investigating the extent to which any mathematical conceptual difficulties may affect students' understanding of associated physics concepts in thermodynamics $[5,6]$. In this area, as with many physics areas, we expect that

Published by the American Physical Society under the terms of the Creative Commons Attribution 3.0 License. Further distribution of this work must maintain attribution to the author(s) and the published article's title, journal citation, and DOI. specific mathematical concepts are required for a complete understanding and appreciation of the physics. Although several of the mathematical concepts we probed are not taught until the third-semester of calculus, a number of those which are essential to junior-level thermal physics (e.g., derivatives, integrals) are also considered essential for calculus-based introductory physics. Our research question in this context is, to what extent can students answer conceptual math questions that appear identical to conceptual physics questions that are stripped of their physical context?

\section{METHODOLOGY}

We sought to probe students' ideas about a few of the mathematical concepts that we expect them to use in the physics classroom. This led to creating questions that looked like physics questions but were simply stripped of their physical context. Since these questions often involve representations that deviate from those typically used in the mathematics domain, we have labeled them "physicsless physics questions" [7].

We have asked physics-less physics questions about integrals and line integrals, based on findings in the context of $P-V$ diagrams $[7,8]$ and about partial differentiation and the product and chain rules based on findings related to material properties in thermodynamics [5,9]. Our findings suggested that these questions were challenging to a significant population of physics students at the upper division.

While these questions were originally developed for physics students, we realized that an interesting comparison, and one that could provide insight on issues of epistemic framing [10], transfer [11-13], and/or disciplinary 
conventions, would be to ask these questions to students in a third-semester calculus course, after all relevant mathematics instruction. Results should capture student thinking about many concepts that had been taught up to that point (e.g., slope, derivative, integration, partial differentiation, etc.). We designed a short written, free-response survey containing only the physics-less questions. This survey has been given to more than 150 students over multiple semesters of the University of Maine's third-semester calculus course, Multivariate Calculus, always in the last week of class.

The data here are not matched across questions. Instructor participation decreased after the first semester, which accounts for the sample size nearly halving from semester 1 to semesters 2 and 3. Many students did not answer every question in the survey, usually due to time constraints, so that for any given semester the numbers are slightly different from one question to the next. We also assume that, on average, the students from the three different semesters are samples drawn from the same population.

\section{ASSESSMENT TASKS AND RESULTS}

Given our results on other physics-less physics questions in physics classes, we did not want to make any assumptions about multivariable calculus students' understanding of slope and (single-variable) derivatives. Therefore, we included physics-less physics questions about concepts of slope and derivative on our survey in order to shed additional light on student thinking regarding these concepts without the burden of the physics context. The result was

$f$ is a function of the variable $x$, i.e. $f=f(x)$. Consider the graph of $f(x)$ versus $x$ shown on the right.

Rank the value (NOT absolute value!) of the slope of $f(x)$ at each of the four values of $x$ (i.e., $a, b, c, d$ ) from greatest to least. Keep in mind that positive values are greater than negative values, and that a larger negative value is less than a smaller negative value. If two slopes are equal, state this explicitly. If there is not enough information to decide, state so explicitly. Explain your reasoning.

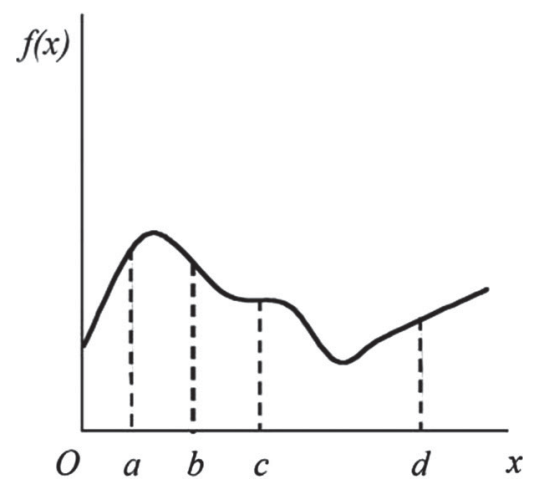

FIG. 1. Slope Ranking Task.
TABLE I. Student performance on slope ranking task, by semester of administration.

\begin{tabular}{lc}
\hline \hline & Slope of $f(x)$ at $(a>d>c>b)$ \\
\hline Semester 1 $(N=70)$ & $79 \%$ \\
Semester 2 $(N=31)$ & $90 \%$ \\
Semester 3 $(N=37)$ & $89 \%$ \\
\hline \hline
\end{tabular}

two questions that are effectively physics-less versions of questions from the PER literature: the Slope Ranking Task and the Derivative Sign and Ranking Task.

\section{A. Slope Ranking Task}

In the Slope Ranking Task (Fig. 1), students are asked to order the slope of the drawn function at four different values of $x$. In other words, we wanted students to identify the value of the instantaneous slope at each point. The question attempts to dissuade ranking absolute values and contains a great deal of language explaining the desired form of the response. A correct response on the Slope Ranking Task requires students to associate slope with the steepness of the tangent line of the curve at the four given points.

\section{Difficulties in mathematics consistent with physics difficulties among introductory students}

Over three semesters, roughly $85 \%$ of students were able to complete this task successfully (see Table I). Fewer than half of the students with correct rankings provided any reasoning for their ranking. Any mention of steepness of the curve, or the slope at the curve at the points, was considered a correct explanation. We do not presume that this is the full extent of students who are able to explain this response, but rather that many students simply did not write anything down.

Although the incidence of incorrect rankings was small (roughly 15\%), a few commonalities could be determined, though none of them accounted for more than 5\% of the total responses. The most common incorrect response was a ranking that is consistent with the average slope between points rather than the instantaneous slope at a point. A line segment drawn between the values of the function from points 0 to $a$ would have a larger slope than a line segment drawn between the values from $c$ to $d$. (See Fig. 2 for a sample response of this nature.)

This type of confusion-students interchanging average and instantaneous velocity for objects that are

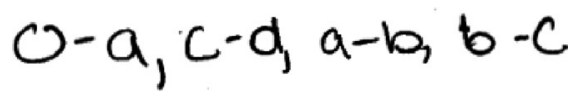

FIG. 2. Student response from the Slope Ranking Task indicating a ranking consistent with the average slope between points rather than the instantaneous slope at each point. 
not experiencing constant acceleration-has been documented in the PER literature among first-semester calculus-based physics students in the context of kinematics $[4,14]$.

The second most common incorrect response given was a ranking consistent with the value of the function at each point rather than the slope at each point. This confusion has been previously reported in the PER literature by Beichner in the context of graphical interpretation of kinematics [4]. It is unclear to what extent the errors reported by Beichner may occur due to student confusion with the mathematics rather than the physical context, but it seems highly plausible that some students are struggling simply with the mathematics implicit in the question.

While only a few students $(\sim 5 \%)$ in the population of third-semester calculus enrollees are making this kind of error, it seems reasonable that students exiting a firstsemester course might be displaying similar or increased difficulty. We are currently exploring the extent of these difficulties among introductory students to further illustrate these findings.

\section{B. Derivative Sign and Ranking Task}

The Derivative Sign and Ranking Task (Fig. 3) asks students to determine the signs of and compare the magnitudes of the derivatives of three different functions of the independent variable $x$ at the same value of $x$, based on a set of graphs of the three functions. This question requires students to make a connection between a derivative and either the slope or the change in the function, which must then be interpreted from the graph. This task was specifically written to overlay the assessed concept in the previous question, while also identifying students who could rank the slopes of a line but might not be able to connect "derivative" with the slope of the line.

The curves of the three functions were drawn to allow common incorrect reasoning to be more clearly determinable. We found that more than half of the students were able to state that the derivatives for all three functions were positive (see Table II). Because of the potentially mislead-
Three functions have been graphed on the graph shown at right [below], and are labeled $f(x), g(x)$, and $h(x)$.

$$
\text { Consider the derivatives }\left.\frac{d f}{d x}\right|_{x=a},\left.\frac{d g}{d x}\right|_{x=a} \text {, and }\left.\frac{d h}{d x}\right|_{x=a} \text {. }
$$

a) For each of the derivatives listed above, state whether the derivative is positive, negative, zero, or there is not enough information to decide. Please explain your reasoning.

b) Rank the absolute values of the three derivatives using the following possible terms: greater than or equal. If there is not enough information to decide, please say so explicitly. Explain your reasoning.

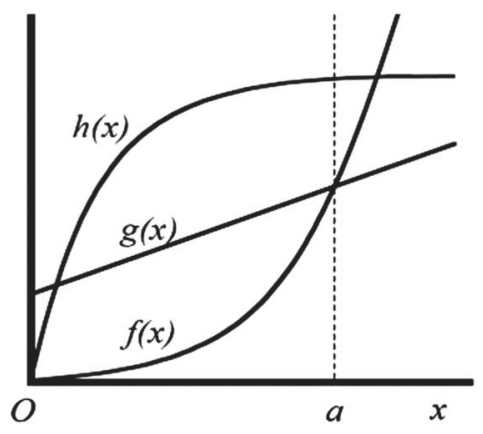

FIG. 3. Original Derivative Sign and Ranking Task. [Later versions featured a curve with a more clearly positive slope for $h(x)$ at $x=a$; see Fig. 5.]

ing flatness of curve $h(x)$, some students stated the derivative at $x=a$ was zero or not determinable, which we allowed as correct in our analysis.

A curve with a more clearly positive slope was added in place of $h(x)$ after the first administration, which seemed to effectively eliminate this "alternative" correct explanation.

\section{Results of the derivative tasks}

The responses of signs and ranking tasks were fairly consistent, and most student reasoning was easily inferred from the paired set of responses. The most common

TABLE II. Response distribution for Derivative Sign and Ranking Task. Percentage in the "All correct responses" row are the sum of the "Preferred" and "Alternative correct responses"; 2nd derivative responses are those students whose sign choice is consistent with that of the 2 nd derivative, but not necessarily identified by the student as a 2 nd derivative response.

\begin{tabular}{lccc}
\hline \hline & $\begin{array}{c}\text { Semester } 1(N=82) \\
\text { Original version }\end{array}$ & $\begin{array}{c}\text { Semester 2 }(N=34) \\
\text { Modified version }\end{array}$ & $77 \%$ \\
\hline All correct responses & $71 \%$ & $62 \%$ & $69 \%$ \\
$\quad \begin{array}{l}\text { Preferred correct response } \\
d f / d x, d g / d x, d h / d x \text { are positive }\end{array}$ & $51 \%$ & $62 \%$ & $8 \%$ \\
$\quad \begin{array}{l}\text { Alternative correct response } \\
d f / d x, d g / d x \text { are positive; } d h / d x \\
\text { is zero or not enough information }\end{array}$ & $20 \%$ & & $8 \%$ \\
2nd derivative response & & $18 \%$ & $13 \%$ \\
\hline \hline
\end{tabular}




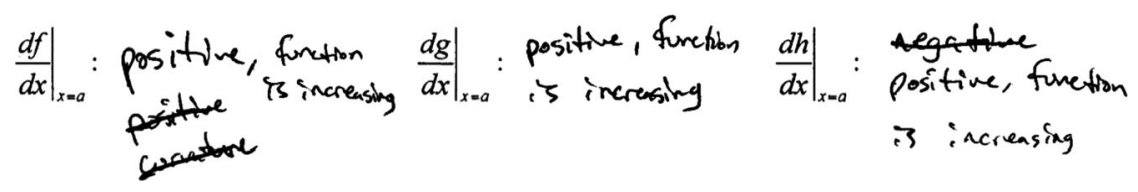

FIG. 4. Example of student response to derivative sign task that started to use 2nd derivative reasoning-note the "positive curvature" in the response-but switched to the correct reasoning.

incorrect response (7\% of all responses) on the original version was a set of signs and a ranking consistent with that for the values of the 2 nd derivatives of these curves. A student categorized as such would give the signs as (positive, zero, and negative) for the derivatives at $f(a), g(a)$, and $h(a)$, respectively. This is consistent with the rate of change of the slope of the function at $x=a$ rather than the rate of change of the function itself. Most responses in this category had very little, if any, accompanying reasoning. One student did seem to correct himself in the middle of his response, using "curvature" to justify his signs (see Fig. 4), and then switching to reasoning about the function(s) "increasing" to decide about the derivative.

We were concerned that the question wording may have been unclear. The first version stated, "For each of the derivatives listed above, state whether the derivative is positive, negative, zero or there is not enough information to decide." In response, we altered the language to indicate more clearly that we sought the signs of the derivatives of the functions and gave the expressions for the derivatives in the response area for emphasis (Fig. 5). However, the semester 2 students gave 2 nd derivative responses at the much higher rate of $18 \%$ (the rate in semester 3 was 13\%) with the modified version (see Table II), implying that the question wording and presentation were not the issue, and that this is still a significant difficulty for students even after multivariable calculus.

\section{2nd derivative responses are not likely to be an issue of reading the slope of the curve}

We can cast additional light on the thinking of those students that gave responses consistent with 2 nd derivative reasoning by examining their responses to the Slope Ranking Task.

Nearly all $(95 \%)$ of those students who gave a $2 n d$ derivative response on the derivative tasks gave a correct ranking on the Slope Ranking Task. This suggests that these students are able to make sense of the slope of a curved surface, but do not match the idea of derivative with the instantaneous slope of the curve. One possible explanation (among many) would be that a student might carry two notions of derivative: that of the rate of change of the function and that of the slope of the function. Students may use one notion or the other as they see fit in a given context, but may, at times, use them simultaneously. Thus, a question about derivative may cause them to think of the "change in the slope" and give a 2nd derivative response. Additional research would be necessary to fully reveal this phenomenon.

Analysis of the responses to the derivative ranking portion of the task [see part (b) in Fig. 5] revealed only one noteworthy result. Most students who gave a correct sign for the derivatives in part (a) correctly ranked the values of the derivatives in part (b). Among those responses that had all positive in part (a), there were some instances $(<5 \%)$

Three functions have been graphed on the graph shown at right, and are labeled $f(x), g(x)$, and $h(x)$.

Consider the derivatives $\left.\frac{d f}{d x}\right|_{x=a},\left.\frac{d g}{d x}\right|_{x=a}$, and $\left.\frac{d h}{d x}\right|_{x=a}$

a) State whether the derivatives listed above (specifically at the value of $x=a$ ) are positive, negative, zero, or there is not enough information to decide. Please explain your reasoning.
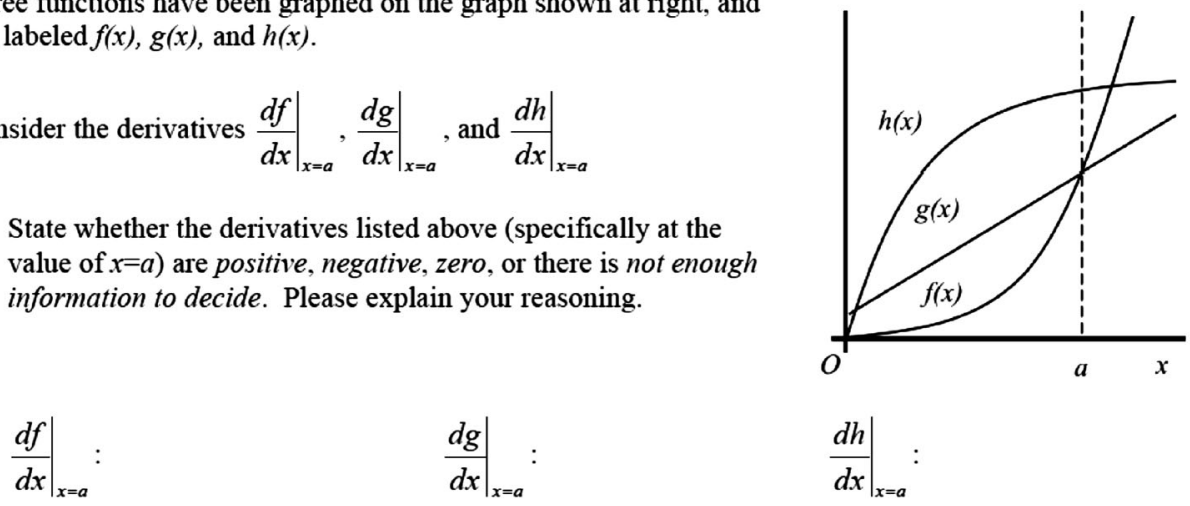

b) Consider the absolute values of the three derivatives at $x=a$. Put them in order from least to greatest.If there is not enough information to decide, please say so explicitly. Explain your reasoning.

FIG. 5. Modified Derivative Sign and Ranking Task, to have more explicit wording and a greater slope for $h(x)$ at $x=a$. 
of student rankings that were consistent with a ranking of the areas under the curves of the functions, with at least two instances of students explicitly justifying their answers as "areas under the curve." If this question were given in a kinematics context, researchers would likely identify student confusion with the associated kinematics quantities; instead, this physics-less physics question points to at least a few students who use notions about area to answer questions about derivatives independent of physical context.

\section{CONCLUSIONS}

Preliminary results from questions about slopes and derivatives administered in a multivariable calculus course suggest that students have difficulties conceptualizing mathematics tasks that are common to the ways in which we ask questions in physics courses. There is a growing body of work on transfer [11-13], with findings that students have difficulties transferring mathematical ideas across disciplines. The type of mathematical tasks we want our students to do in a physics class may simply be foreign to their mathematical ways of thinking. Some of their demonstrated difficulties seem to have origins in the understanding of the math concepts themselves. These results are consistent with results from questions about integrals in thermodynamics contexts [8]. This aspect of our results will be explored further in future research. We are continuing to collect and analyze data from written questions with an eye toward expanding the scope of our investigation to additional populations at the introductory level, both in physics and in mathematics.

\section{ACKNOWLEDGMENTS}

We gratefully acknowledge the contributions of Brandon Bucy, Evan Pollock, and Donald Mountcastle to this and supporting work, including fruitful discussions. We also acknowledge Trevor Smith for his thorough reading and suggestions on this text, and the thoughtful comments of three reviewers. This work was partially supported by NSF Grants No. DRL-0633951 and No. DUE-0817282, and the Maine Academic Prominence Initiative (WMC).
[1] D. E. Trowbridge and L. C. McDermott, Investigation of student understanding of the concept of velocity in one dimension, Am. J. Phys. 48, 1020 (1980).

[2] D. E. Trowbridge and L. C. McDermott, Investigation of student understanding of the concept of acceleration in one dimension, Am. J. Phys. 49, 242 (1981).

[3] L. C. McDermott, M. L. Rosenquist, and E. H. van Zee, Student difficulties in connecting graphs and physics: Examples from kinematics, Am. J. Phys. 55, 503 (1987).

[4] R. J. Beichner, Testing student interpretation of kinematics graphs, Am. J. Phys. 62, 750 (1994).

[5] B. R. Bucy, J.R. Thompson, and D. B. Mountcastle, Student (mis)application of partial differentiation to material properties, AIP Conf. Proc. 883, 157 (2007).

[6] D. B. Mountcastle, B.R. Bucy, and J.R. Thompson, Student estimates of probability and uncertainty in advanced laboratory and statistical physics courses, AIP Conf. Proc. 951, 152 (2007).

[7] W. M. Christensen and J. R. Thompson, Investigating student understanding of physics concepts and the underlying calculus concepts in thermodynamics, in Proceedings of the 13th Annual Conference on Research in
Undergraduate Mathematics Education (Mathematical Association of America, Oberlin, OH, 2010).

[8] E. B. Pollock, J.R. Thompson, and D. B. Mountcastle, Student understanding of the physics and mathematics of process variables in $P-V$ diagrams, AIP Conf. Proc. 951, 168 (2007).

[9] J.R. Thompson, B. R. Bucy, and D. B. Mountcastle, Assessing student understanding of partial derivatives in thermodynamics, AIP Conf. Proc. 818, 77 (2006).

[10] J. Tuminaro and E. F. Redish, Elements of a cognitive model of physics problem solving: Epistemic games, Phys. Rev. ST Phys. Educ. Res. 3, 020101 (2007).

[11] N.S. Rebello, Can we assess efficiency and innovation in transfer?, AIP Conf. Proc. 1179, 241 (2009).

[12] See Transfer of Learning from A Modern Multidisciplinary Perspective, edited by J. Mestre (Information Age, Greenwich, CT, 2005).

[13] J.F. Wagner, A transfer-in-pieces consideration of the perception of structure in the transfer of learning, J. Learn. Sci. 19, 443 (2010).

[14] P.S. Shaffer and L. C. McDermott, A research-based approach to improving student understanding of the vector nature of kinematical concepts, Am. J. Phys. 73, 921 (2005). 